\title{
新型嘧啶-水杨酸类抑制剂的设计、合成及其生物活性研究
}

\author{
曲仁渝严耀超杨景芳陈琼*杨光富* \\ (华中师范大学化学学院 农药与化学生物学教育部重点实验室 武汉 430079)
}

\begin{abstract}
摘要 乙酰羟酸合成酶(Acetohydroxyacid synthase, AHAS)是一类重要的除草剂靶标, 但是靶向 AHAS 的抑制剂是所有 除草剂类型中抗性最为严重的一类，因此设计具有反抗性的 AHAS 抑制剂显得尤为必要. 本工作基于前期低抗性 AHAS 抑制剂结构, 利用 “构象柔性度分析” 的设计策略合理增加分子柔性，设计并合成了 13 个含 “双氧桥” 结构的 嘧啶水杨酸类抑制剂. 目标分子均经过氢谱 $\left({ }^{1} \mathrm{H} N \mathrm{NM}\right)$ 、碳谱 $\left({ }^{13} \mathrm{C} \mathrm{NMR}\right)$ 和高分辨质谱(HRMS)确证. 酶水平活性结果显 示, 部分化合物对 P197L 突变体表现出了微摩尔水平的抑制作用, 特别是 2-((4,6-二甲氧基嘧啶-2-基)氧基)-6-(2-氟-4硝基苯氧基)-4-甲基苯甲酸(61)对 P197L 突变体表现出了良好的反抗性. 盆栽除草活性结果表明, 个别化合物对抗性杂 草播娘蒿具有一定的除草活性，其中 2-((4,6-二甲氧基嘧啶-2-基)氧基)-6-(2-氟苯氧基)-4-甲基苯甲酸(6b)在 $150 \mathrm{~g}$ ai/ha 浓度下, 对敏感生物型播娘蒿和抗性播娘蒿均达到 $80 \%$ 的除草防效, 具有进一步深入研究的价值.
\end{abstract}

关键词 乙酰差酸合成酶; 反抗性; P197L 突变体; 构象柔性; 抗性杂草

\section{Design, Synthesis and Bioactivity of New Pyrimidyl-salicylate Inhibitors}

\author{
Qu, Renyu Yan, Yaochao Yang, Jingfang Chen, Qiong* Yang, Guangfu* \\ (Key Laboratory of Pesticide and Chemical Biology, College of Chemistry, Central China Normal University, Wuhan 430079)
}

\begin{abstract}
Acetohydroxyacid synthase (AHAS) was one of important herbicidal targets. However, the issue of weed resistance to commercial AHAS inhibitors has become one of the largest obstacles for their application. Therefore, there is a high demand to design new anti-resistance AHAS inhibitors. Herein, based on the reported low resistance AHAS inhibitors, a series of pyrimidyl-salicylates with "double oxygen bridge" utilized the "conformation flexibility analysis" strategy were designed. All the synthesized compounds were characterized by ${ }^{1} \mathrm{H}$ NMR, ${ }^{13} \mathrm{C}$ NMR and HRMS. The bioactivity results showed that most of the derivatives displayed good inhibitory activities against P197L mutant. Especially, 2-((4,6-dimethoxypyrimidin-2yl)oxy)-6-(2-fluoro-4-nitrophenoxy)-4-methylbenzoic acid (6l) was identified as the most potent anti-resistance AHAS inhibitor. In addition, some compounds showed good weed control for resistant Descurainia sophia (P197L AHAS). Most importantly, 2-((4,6-dimethoxypyrimidin-2-yl)oxy)-6-(2-fluorophenoxy)-4-methylbenzoic acid (6b) showed 80\% herbicidal activities against sensitive and resistant Descurainia sophia at the dosage of $150 \mathrm{~g}$ ai/ha. These results indicated that this type of compounds worth of the further investigation.
\end{abstract}

Keywords acetohydroxyacid synthase; anti-resistance; P197L mutant; conformation flexibility; resistant weed

乙酰羟酸合成酶(AHAS, EC 2.2.1.6)是生物体内支 链氨基酸合成的关键酶, 其主要催化一分子丙酮酸以及 一分子 2-羰基丁酸(异亮氨酸的合成途径)或两分子的丙 酮酸(亮氨酸和缅氨酸的合成途径)分别合成产物 $\alpha$-乙酰 羟基丁酸和 $\alpha$-乙酰乳酸 ${ }^{11}$. 由于 AHAS 在植物体内独特 的生理功能, 早在 20 世纪 70 年代其已经被发展成为一
类重要的除草剂靶标. 自跨世纪除草剂“氯磺隆”问世以 来, 以 AHAS 为靶标的除草剂品种层出不穷, 截止目前 为止共有五种不同结构类型的抑制剂分别靶向于 AHAS, 包括磺酰䐂类(SUs)、三唑并嘧啶磺酰胺类 (TPs)、嘧啶(硫代)苯甲酸(PTBs)、咪唑啉酮类(IMIs) 以 及三唑啉酮类 $(\mathrm{SCTs})^{[2]}$. 在实际生产中, 已商品化的

\footnotetext{
* Corresponding authors. E-mail: gfyang@mail.ccnu.edu.cn; qchen@mail.ccnu.edu.cn Received January 26, 2019; revised March 4, 2019; published online March 21, 2019. Dedicated to the 100th anniversary of the birth of Professor Ruyu Chen.

Project supported by the National Key Research and Development Program of China (No. 2018YFD0200102) and the National Natural Science Foundation of China (Nos. 21837001. 21772058).

国家重点研发计划(No. 2018YFD0200102)、国家自然科学基金(Nos. 21837001.21772058)资助项目.
} 
AHAS 除草剂往往具有超高效的除草活性、良好的作物 选择性以及对哺乳动物高度安全等优点, 然而由于该类 除草剂在田间被大面积连续使用, 对杂草造成的巨大选 择压力致使其抗性问题日益突出 ${ }^{[3]}$. 根据除草剂抗性委 员会(HRAC, Herbicide resistance action committee)截止 2018 年的统计报道, AHAS 抑制类除草剂的抗性杂草物 种数高达 160 余种, 是所有除草剂类型中抗性最为严重 的一类 ${ }^{[4]}$. 因此, 发展具有全新反抗性机制且保持原有 产品优势的 AHAS 抑制剂先导结构迫在眉睫!

近些年的研究表明, 抑制剂分子与靶酶 AHAS 催化 亚基的结合位点发生氨基酸残基的单点突变, 导致相互 作用力减弱, 是造成杂草对 AHAS 抑制剂产生高倍抗性 问题的主要原因. 目前已知的抗性突变位点有 7 个, 分 别是 Ala122、Pro197、Ala205、Asp376、Trp574、Ser653、 Gly654(以 Arabidopsis thaliana, AtAHAS 催化亚基序列 进行编号), 它们都位于 AHAS 催化亚基的 5 个高度保守 区域 ${ }^{[5]}$. 这 7 个突变位点中, Pro-197-Leu 突变引起的杂 草抗性报道最为广泛, 并且该突变会对多种类型 AHAS 抑制剂产生严重的交互抗性 ${ }^{[6]}$. 例如, 杂草 Amaranthus retroflexus 的 AHAS 发生 P197L 突变后，对 SUs、IMIs、 TPs、PTBs 以及 SCTs 五类 AHAS 抑制类除草剂均产生 十倍甚至百倍以上的抗性 ${ }^{[7]}$. 因此, 针对 P197L 突变体 设计一类在酶水平以及活体水平均具有高活性的反抗 性 AHAS 抑制剂具有重要的现实意义. 最近, 我们发现 在酶突变前后抑制剂分子构象柔性变化与抗性倍数高 低之间存在着一定的关联性, 并提出一种基于 “构象柔 性度分析” (conformational flexibility analysis)的反抗性

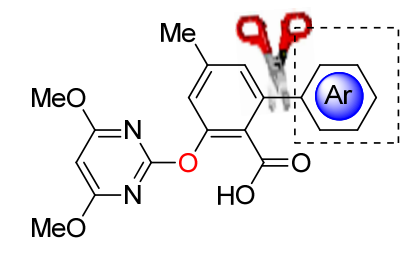

Pyrimidine-biphenyl hybrid

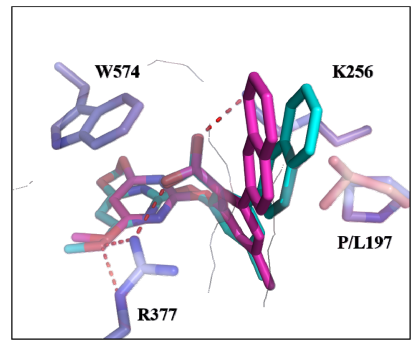

Compound A, Ar = 2-naphthyl
分子设计策略 ${ }^{[8]}$. 基于该策略, 我们前期发展了一种通 过柔性桥链连接的嘧啶一联芳基类衍生物，代表性化合 物 A 对野生型 AtAHAS 和 P197L 突变体均表现出良好 的酶抑制作用 ${ }^{[9]}$. 但令人遗憾的是, 虽然其对 P197L 突 变体的抗性倍数(约 19 倍)相较于 SUs、TPs 类 AHAS 抑 制剂(>500 倍)有大幅下降，但仍然没有达到反抗性的 设计要求. 因此根据上述反抗性药物分子设计理念, 继 续增加配体分子的构象柔性，使其更加适应突变体空 腔，增强对突变体的抑制活性，实现抑制剂对 P197L 突 变体的反抗性.

基于此，我们将低抗性 AHAS 抑制剂 $\mathbf{A}$ 作为基本骨 架，侧链引入苯氧基结构，设计了一类含 “双柔性氧桥” 的新型 AHAS 抑制剂结构(图 1). 从分子对接叠合图中, 我们发现母体分子 $6 \mathrm{a}$ 在酶突变前后的构象翻转相比于 化合物 A 更加显著，可能更有利于抑制剂与 P197L 突变 体结合以及反抗性 AHAS 抑制剂的发现. 而后我们合成 了一系列的相关衍生结构 $6 \mathrm{~b} \sim 6 \mathrm{~m}$, 所有目标分子结构 均经过 ${ }^{1} \mathrm{H}$ NMR, ${ }^{13} \mathrm{C}$ NMR 以及高分辨质谱确定，同时 测试了它们对野生型 AtAHAS 和 P197L 突变体的酶抑制 活性，并利用分子模拟探讨了该系列分子的反抗性机 制. 此外也将它们对敏感型和抗性(P197L-AHAS)杂草 播娘蒿的除草活性进行了评价.

\section{1 结果与讨论}

\section{1 目标化合物 $6 a \sim 6 m$ 的合成}

1.1.1中间体 $\mathbf{3}$ 的合成 在合成目标分子的过程中，我们以 3,5-二羟基甲苯

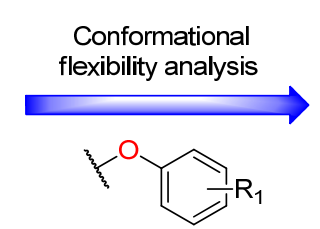

Structural optimization

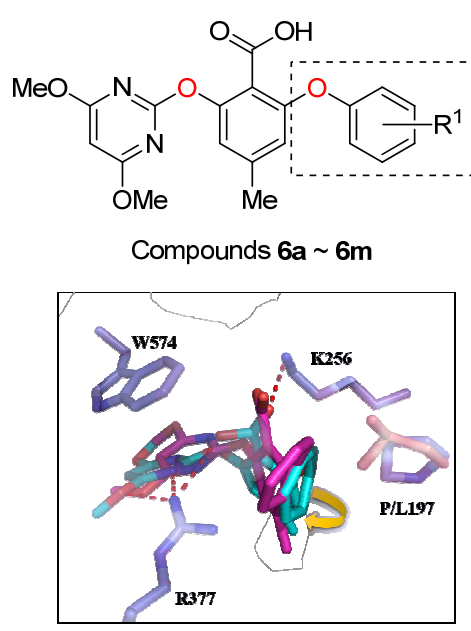

Compound $6 a, \mathrm{R}^{1}=\mathrm{H}$

图 1 目标化合物 6 的分子设计以及代表性化合物 A 和 $6 \mathbf{a}$ 在野生型 AtAHAS 和 P197L 突变体中的叠合图

Figure 1 Design of target compounds 6, and simulated binding modes overlay of representative compounds $\mathbf{A}$ and $\mathbf{6 a}$ with wild-type AtAHAS and its P197L mutant, respectively

The green molecule represent binding mode between ligand and wild-type AtAHAS, and the pink molecule represent binding mode between ligand and P197L mutant 
(苔黑酚, 化合物 $\mathbf{1}$ ) 作为起始原料, 首先在通 $\mathrm{CO}_{2}$ 的条件 下, 将化合物 $\mathbf{1}$ 的 2 号位引入重要的药效羧基片段, 经 过浓盐酸酸化可以合成中间体 2. 随后如何在双氧桥的 两个侧链连接不同的芳香片段是合成的关键, 因此我们 利用类缩醛保护策略, 将中间体 2 与丙酮在二氯亚砜和 DMAP 的作用下进行环化保护反应, 同时保护羧基以及 其中的一个羟基得到了关键中间体 $\mathbf{3}^{[10]}$, 以便提高后续 的反应选择性.

\subsection{2中间体 $\mathbf{4}$ 和 $\mathbf{5}$ 的合成}

为了利于后续底物扩展, 我们主要利用两种合成方 法对右侧的二芳基醚片段进行构建. 第一种, 利用二芳 基碘鎓四氟嗍酸盐与中间体 3 中酚羟基在叔丁醇钾的作 用下发生反应合成中间体 $\mathbf{4}$, 对称的二芳基碘鎓盐是利 用文献报道的方法进行合成 ${ }^{[11]}$; 第二种, 利用 $S_{N} A r$ 亲 核取代反应, 将中间体 3 与带有强吸电子基团的(取代) 对氟硝基苯或邻氟硝基苯在碳酸钾的作用下, 得到了含 有硝基取代的中间体 4. 最后在 $\mathrm{NaOH}$ 的作用下将类缩 醛结构水解, 酸化后可不经纯化直接得到 6-苯氧基水杨 酸中间体 $\mathbf{5}$.

\subsection{3 化合物 $\mathbf{6 a} \sim 6 \mathbf{6 m}$ 合成}

在足量碳酸钾的作用下, 将上一步得到的中间体 $\mathbf{5}$ 与 4,6-二甲氧基-2-甲砜基嘧啶(DMSP)溶解于最适反应 溶剂甲苯中进行 $\mathrm{S}_{\mathrm{N}} \mathrm{Ar}$ 取代反应, 原料反应完全后脱干 甲苯, 酸化即可得到粗产品, 后经柱层析提纯即可得到 对应目标分子 $6 \mathbf{a} \sim 6 \mathrm{~m}$, 收率均在 $70 \%$ 以上(Scheme 1).

\section{2 抑制活性以及反抗性机制研究}

通过前期报道的测试方法 ${ }^{[8]}$, 针对野生型 AtAHAS
和 P197L 突变体，分别对已合成的化合物 $6 \mathbf{a} \sim 6 \mathbf{m}$ 进行 了酶抑制动力学常数 ( $K_{\mathrm{i}}$ 值)的测定. 将化合物对 $\mathrm{P} 197 \mathrm{~L}$ 突变体的 $K_{\mathrm{i}}$ 值与其对野生型 AtAHAS 的比值定义为抑 制剂对 P197L 突变体的抗性倍数(RF 值).

该系列化合物的酶水平抑制活性结果如表 1 所示, 其中对照药剂氯磺隆、唑嘧磺草胺对野生型 AtAHAS 均 表现出优异的抑制作用, 但它们对 P197L 突变体的抑制 活性仅有 103 和 $25.8 \mu \mathrm{mol} \cdot \mathrm{L}^{-1}$, 抗性倍数高达 2060 倍 和 68 倍, 这也进一步从酶学水平解释了这两个商品化 AHAS 抑制剂高抗的原因. 而前期合成的先导化合物 A 对野生型 AtAHAS 和 P197L 突变体均表现出微摩尔级别 的抑制活性 $\left(K_{\mathrm{i}, \text { wild-type }}=0.25 \mu \mathrm{mol} \cdot \mathrm{L}^{-1}, K_{\mathrm{i}, \mathrm{P} 197 \mathrm{~L}}=4.81\right.$ $\left.\mu \mathrm{mol} \cdot \mathrm{L}^{-1}\right)$, 其抗性倍数相比于氯磺隆有接近 107 倍下 降, 呈现出低抗性水平. 令人欣喜的是, 本文通过增加 侧链构象柔性所设计的代表性化合物 $\mathbf{6 a}\left(\mathrm{R}^{1}=\mathrm{H}\right)$, 对 P197L 突变体的抗性倍数仅为 1.35 倍, 相较于先导化合 物 A, 其抗性倍数进一步下降近 14 倍, 但该化合物对野 生型 AtAHAS 和 P197L 突变体的抑制活性并不理想 $\left(K_{\mathrm{i}, \text { wild-type }}=9.47 \mu \mathrm{mol} \cdot \mathrm{L}^{-1}, K_{\mathrm{i}, \mathrm{P} 197 \mathrm{~L}}=12.8 \mu \mathrm{mol} \cdot \mathrm{L}^{-1}\right)$. 为 了进一步增加分子对野生型 AtAHAS 和 P197L 突变体的 抑制活性, 并继续降低分子的抗性程度实现反抗性, 我 们合成了 $6 \mathrm{a}$ 的衍生物 $6 \mathrm{~b} \sim 6 \mathrm{~m}$. 观察该系列衍生物的 $K_{\mathrm{i}}$ 值，当分子中 $\mathrm{R}^{1}$ 基团引入 $\mathrm{F} 、 \mathrm{Cl}$ 时，发现化合物的取代 基位置对 P197L 突变体的酶抑制活性影响有一定的规 律: $2-\mathrm{F}>3-\mathrm{F}>4-\mathrm{F}(\mathbf{6} \mathbf{b}>\mathbf{6 c}>\mathbf{6 d}), 2-\mathrm{Cl}>3-\mathrm{Cl}>4-\mathrm{Cl}$ $(6 e>6 f>6 g)$, 且 $R^{1}$ 为 $F$ 取代时化合物对 $P 197 L$ 突变体 的抑制活性明显优于 $\mathrm{Cl}$ 取代, 特别是化合物 $\mathbf{6 b}\left(\mathrm{R}^{1}=\right.$<smiles>Oc1cc(O)cc([Al])c1</smiles>

$\underset{(1) \mathrm{CO}_{2}, \mathrm{KHCO}_{3} \text {, glycerol }}{\longrightarrow}$ (2) conc. $\mathrm{HCl}$

1 $\mathrm{BF}_{4}$<smiles>[R][I-](C)(C)/C=C\C(=C)C</smiles>

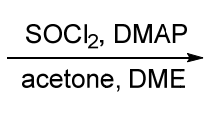<smiles>CC1(C)OC(=O)c2c(O)cc(N)cc2O1</smiles>

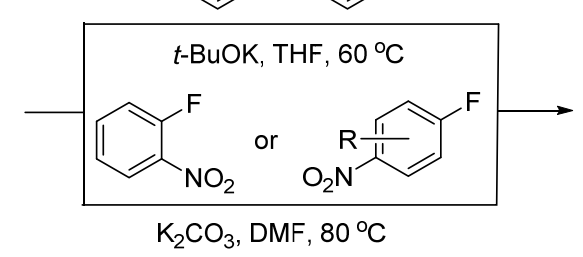<smiles>[R]c1ccc(Oc2cc([N+](=O)[O-])cc3c2C(=O)OC(C)(C)O3)cc1</smiles>

$\frac{\text { (1) } \mathrm{NaOH}, 60^{\circ} \mathrm{C}}{\text { (2) } 2 \mathrm{~mol} / \mathrm{L} \mathrm{HCl} \text { (aq.) }}$<smiles>[R]c1ccc(Oc2cc([N+](=O)[O-])cc(O)c2C(=O)O)cc1</smiles>

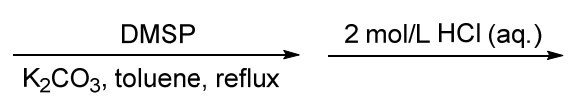<smiles>COc1cc(OC)nc(OC(C)=O)n1</smiles>

图式 1 目标分子 $6 \mathrm{a} \sim 6 \mathrm{~m}$ 的合成路线

Scheme 1 Synthetic route of title compounds $6 \mathbf{6} \sim 6 \mathbf{m}$ 
表 1 目标化合物 6a 6m 对野生型 AtAHAS 和 P197L 突变体的酶水平抑制活性数据以及其对应抗性倍数

Table 1 Inhibitory activities of compounds $\mathbf{6 a} \sim \mathbf{6 m}$ against wild-type AtAHAS and P197L mutant

\begin{tabular}{|c|c|c|c|c|}
\hline \multirow{2}{*}{ 化合物 } & \multirow{2}{*}{$\mathrm{R}^{1}$} & \multicolumn{3}{|c|}{ AtAHAS inhibition constant $K_{\mathrm{i}} /\left(\mu \mathrm{mol} \cdot \mathrm{L}^{-1}\right)$} \\
\hline & & 野生型 AtAHAS & P197L 突变体 & 抗性倍数 $(\mathrm{RF})^{a}$ \\
\hline 6a & $\mathrm{H}$ & $9.47 \pm 1.12$ & $12.80 \pm 2.10$ & 1.35 \\
\hline $6 \mathbf{b}$ & $2-\mathrm{F}$ & $5.87 \pm 0.49$ & $6.34 \pm 0.23$ & 1.08 \\
\hline $6 \mathbf{c}$ & $3-\mathrm{F}$ & $6.03 \pm 1.50$ & $23.90 \pm 8.60$ & 4.40 \\
\hline 6d & $4-\mathrm{F}$ & $6.86 \pm 0.96$ & $54.90 \pm 6.60$ & 8.00 \\
\hline $6 e$ & $2-\mathrm{Cl}$ & $17.20 \pm 2.80$ & $21.50 \pm 3.70$ & 1.25 \\
\hline $6 f$ & $3-\mathrm{Cl}$ & $5.55 \pm 0.60$ & $26.00 \pm 4.60$ & 13.47 \\
\hline $6 \mathrm{~g}$ & $4-\mathrm{Cl}$ & $9.13 \pm 0.93$ & $35.90 \pm 6.60$ & 3.93 \\
\hline $6 h$ & $2-\mathrm{NO}_{2}$ & $2.82 \pm 0.32$ & $29.60 \pm 3.20$ & 10.50 \\
\hline $6 \mathbf{i}$ & $4-\mathrm{NO}_{2}$ & $2.25 \pm 0.22$ & $5.25 \pm 0.39$ & 2.33 \\
\hline $6 \mathbf{j}$ & 3-Me-4-NO ${ }_{2}$ & $10.30 \pm 1.10$ & $22.80 \pm 2.10$ & 2.21 \\
\hline $6 \mathbf{k}$ & $2-\mathrm{Me}-4-\mathrm{NO}_{2}$ & $13.10 \pm 1.50$ & $47.50 \pm 6.80$ & 2.06 \\
\hline 61 & $2-\mathrm{F}-4-\mathrm{NO}_{2}$ & $2.90 \pm 0.42$ & $2.82 \pm 0.32$ & 0.97 \\
\hline $6 \mathrm{~m}$ & $2-\mathrm{Cl}-4-\mathrm{NO}_{2}$ & $5.94 \pm 0.79$ & $21.80 \pm 3.10$ & 3.67 \\
\hline $\mathbf{A}$ & & $0.25 \pm 0.10$ & $4.81 \pm 0.32$ & 19.2 \\
\hline 氯磺隆 ${ }^{b}$ & & $0.05 \pm 0.01$ & $103.00 \pm 14.00$ & 2060 \\
\hline 唑嘧磺草胺 ${ }^{b}$ & & $0.38 \pm 0.26$ & $25.80 \pm 3.20$ & 68.0 \\
\hline
\end{tabular}

${ }^{a} \mathrm{RF}$, Resistance Farctor $=K_{\mathrm{i}, \mathrm{P} 197 \mathrm{~L}} / K_{\mathrm{i} \text {, wild-type; }}{ }^{b}$ 对照药剂: 商品化 AHAS 抑制剂.

$2-\mathrm{F}$ ), 对 P197L 突变体的抑制活性达到 $6.34 \mu \mathrm{mol} \cdot \mathrm{L}^{-1}$, 此 外该化合物对野生型 AtAHAS 也表现出了不错的抑制 作用 $\left(K_{\mathrm{i}, \text { wild-type }}=5.87 \mu \mathrm{mol} \cdot \mathrm{L}^{-1}\right)$, 其抗性倍数仅为 1.08 倍, 相较于未取代的化合物 $\mathbf{6 a}$, 抗性程度进一步降低. 当继续考察取代基对活性影响时, 发现 $\mathrm{R}^{1}$ 为 $4-\mathrm{NO}_{2}$ 取 代, 相应化合物 6i 对 P197L 突变体和野生型 AtAHAS 的 $K_{\mathrm{i}}$ 值均表现出优于化合物 $\mathbf{6 b}$ 的结果, 但其抗性倍数 高于化合物 6b. 为了综合二者优势, 继续在侧链的苯环 上引入双取代基, 发现化合物 $\mathbf{6 j}\left(\mathrm{R}^{1}=3-\mathrm{Me}-4-\mathrm{NO}_{2}\right) 、 \mathbf{6 k}$ $\left(\mathrm{R}^{1}=2-\mathrm{Me}-4-\mathrm{NO}_{2}\right)$ 和 $\mathbf{6 m}\left(\mathrm{R}^{1}=2-\mathrm{Cl}-4-\mathrm{NO}_{2}\right)$ 相较于化合 物 6i, 它们对 P197L 突变体的酶抑制活性均有大幅度的 下降. 但当 $\mathrm{R}^{1}$ 基团为 2-F-4- $\mathrm{NO}_{2}$ 取代时，相应化合物 61 不仅对 $\mathrm{P} 197 \mathrm{~L}$ 突变体的抑制活性 $\left(K_{\mathrm{i}, \mathrm{P} 197 \mathrm{~L}}=2.82 \mu \mathrm{mol} \cdot \mathrm{L}^{-}\right.$ $\left.{ }^{1}\right)$ 优于化合物 $6 \mathbf{i}\left(\mathrm{R}^{1}=4-\mathrm{NO}_{2}\right)$, 且对野生型 AtAHAS 的抑 制活性也达到了微摩尔水平, 与化合物 $\mathbf{6 i}$ 相当. 值得注 意的是, 该化合物对 P197L 突变体的抗性倍数比化合物 6b $\left(R^{1}=2-F\right)$ 更低, RF 值仅有 0.97 倍, 相比于对照药剂 氯磺隆和唑嘧磺草胺, 其抗性倍数分别下降了 2100 倍 和 70 倍, 说明该化合物对 P197L 突变体具有良好的反 抗性.

为了进一步解释化合物 $\mathbf{6 1}$ 的反抗性分子机制, 我们 采用分子对接方法研究化合物与靶酶之间的作用模式. 代表性化合物 61 与野生型和突变型 AHAS 的结合模式 如图 2A 和 2B 所示, 对比化合物 $6 \mathbf{1}$ 在野生型 AtAHAS 和 P197L 突变体的结合模式, 我们发现分子的嘧啶环与 两个活性空腔中的氨基酸残基 W574 均形成了较好的 $\pi-\pi$ 相互作用, 羧基片段和分子末端的硝基则与周围的 氨基酸有良好的氢键作用, 上述的作用力保证了分子无
论在野生型和突变型 AHAS 中均有较好的结合力, 因而 化合物 61 表现出了较高的酶抑制活性. 进一步研究发 现, 当氨基酸残基 P197 突变为 L197 时, 残基位阻增大, 而分子本身具有较大的柔性, 因此通过构象的翻转避免 了末端芳环与 L197 之间的立体冲突. 虽然分子羧基在 突变体中丢失了与 K256 之间的氢键作用, 但是新的结 合构象却形成了更多的相互作用力, 例如, 羧基与 S635、R377 之间以及苯氧基中的氧桥与 K256 之间形成 新的氢键作用, 使小分子与 P197L 突变体更好地结合, 这一定程度上解释了该化合物的反抗性分子机制，也进 一步证明了本文设计的具有柔性构象 AHAS 抑制剂确 实有助于降低其对 P197L 突变体的抗性倍数. 此外, 化 合物 61 相比于其他衍生物, 其分子 4 位硝基与 R199 之 间以及 2 位氟原子与 $\mathrm{K} 256$ 之间可以形成额外的氢键作 用, 这也解释了化合物 61 对 P197L 突变体显示出最优抑 制活性的原因.

\section{3 温室盆栽除草活性研究}

在剂量为 $150 \mathrm{~g}$ ai $/ \mathrm{ha}$ 条件下, 温室盆栽除草活性结 果如表 2 所示, 其中对照药剂唑嘧磺草胺对敏感生物型 播娘蒿表现出完全的抑制作用，但对于抗性生物型播娘 蒿却仅有 $60 \%$ 的除草防效, 表现出了明显的杂草抗性, 与表 1 中的酶水平呈现的抗性倍数相符. 观察本文所合 成化合物 6a $\sim 6 \mathrm{~m}$ 的除草活性, 我们发现大部分的化合 物对敏感生物型播娘蒿具有一定的抑制作用. 其中化合 物 6b、6e、6h 和 61 对敏感生物型杂草播娘蒿表现出超 过 70\%的除草活性. 此外, 个别化合物对抗性播娘蒿表 

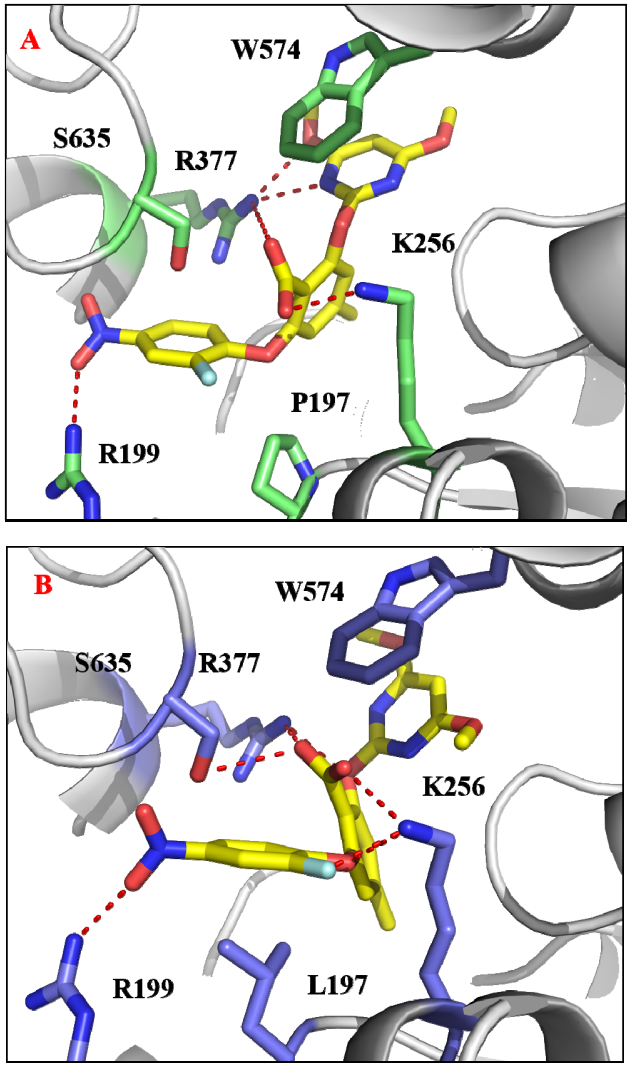

图 2 (A)化合物 61 与野生型 AtAHAS 结合模式示意图以及(B) 化合物 61 与 $\mathrm{P} 197 \mathrm{~L}$ 突变体结合模式示意图

Figure 2 (A) Binding mode of compound $\mathbf{6 1}$ with wild-type AtAHAS and (B) binding mode of compound 61 with P197L mu$\operatorname{tant}$

表 2 目标化合物 $6 \mathrm{a} \sim 6 \mathrm{~m}$ 对敏感生物型播娘蒿以及抗性播 娘蒿的除草活性(生长抑制率/\%)

Table 2 Herbicidal activities of compounds $6 \mathbf{a} \sim 6 \mathbf{m}$ against sensitive and resistant Descurainia sophia (growth inhibitory rate $/ \%)$

\begin{tabular}{ccc}
\hline 化合物 & 敏感型播娘蒿 & 抗性播娘蒿 $^{a}$ \\
\hline $\mathbf{6 a}$ & 50 & 50 \\
$\mathbf{6 b}$ & 80 & 80 \\
$\mathbf{6 c}$ & 60 & 30 \\
$\mathbf{6 d}$ & 40 & 0 \\
$\mathbf{6 e}$ & 70 & 70 \\
$\mathbf{6 f}$ & 50 & 40 \\
$\mathbf{6 g}$ & 0 & 0 \\
$\mathbf{6 h}$ & 70 & 20 \\
$\mathbf{6 i}$ & 30 & 30 \\
$\mathbf{6 j}$ & 0 & 60 \\
$\mathbf{6 k}$ & 30 & 30 \\
$\mathbf{6 1}$ & 80 & 70 \\
$\mathbf{6 m}$ & 40 & 40 \\
唑嘧磺草胺 $^{b}$ & 100 & 60
\end{tabular}

${ }^{a}$ AHAS 突变位点 P197L; ${ }^{b}$ 对照药剂: 商品化 AHAS 抑制剂.

现出了较好的抑制作用, 化合物 $6 \mathrm{~b} 、 6 \mathrm{e}$ 和 61 对抗性播 娘蒿防效超过 70\%，除草活性优于商品化 AHAS 抑制剂
唑嘧磺草胺. 综合以上数据, 进一步发现化合物 $6 \mathrm{~b}$ 不仅 表现出了活体反抗性, 并且对敏感生物型和抗性播娘蒿 的除草活性达到 $80 \%$, 具有进一步深入研究的价值. 因 此，本文的分子设计策略具有一定可行性，有望设计出 具有更高除草活性的反抗性 AHAS 抑制剂.

\section{2 结论}

本文基于 “构象柔性度分析” 分子设计策略，将先 导化合物 $\mathbf{A}$ 作为基本骨架, 增加侧链构象柔性, 引入苯 氧基片段，设计了一类具有“双柔性氧桥”的新型 AHAS 抑制剂. 以苔黑酚作为起始原料，经过羧基化、环化、 $\mathrm{S}_{\mathrm{N}} \mathrm{Ar}$ 亲核取代反应等 5 步反应，以中等偏上的收率合成 了目标化合物 $6 \mathrm{a} \sim 6 \mathrm{~m}$. 采用分子对接的方法, 对比代 表性化合物 6a 在酶突变体后的结合构象, 发现在 P197L 突变体中, 分子相比于其在野生型靶酶构象有较大翻 转, 避免了与残基 L197 之间的立体冲突, 从而降低抗 性程度. 经过后续的衍生化, 发现绝大多数衍生物对 P197L 突变体的抗性倍数维持在 0.9 至 10.0 范围内, 特 别是化合物 61, 不仅对野生型 AtAHAS 和 P197L 突变体 表现出较优的抑制活性, 并且抗性倍数仅有 0.97 , 具有 良好的反抗性. 同时在盆栽除草活性篮选中，化合物 6b、6e 和 61 对抗性播娘蒿的防效超过 $70 \%$, 优于对照 药剂唑嘧磺草胺. 因此, 该类 AHAS 抑制剂具有进一步 研究的潜力, 并为新型反抗性 AHAS 抑制剂的设计提供 了新的先导骨架.

\section{3 实验部分}

\section{1 仪器与试剂}

MR 3001 型恒温磁力搅拌器(Heidolph, German), R-200 型旋转蒸发仪(Büchi, Flawil, Switzerland), Sartotuis 万分之一电子天平, ZF-20D 暗箱式紫外分析仪, SHZ-D(III)循环水式真空原, WS70-1 型远红外线干燥箱 等仪器. ${ }^{1} \mathrm{H}$ NMR 和 ${ }^{13} \mathrm{C}$ NMR 使用 Varian VNMR 400 $\mathrm{MHz}$ 和 $600 \mathrm{MHz}$ 核磁共振仪(Varian, Palo Alto, CA)测 定, 以 TMS 作为内标, 以気代氯仿、氛代二甲亚砜等为 溶剂; 质谱和高分辨质谱数据测定分别使用 DSQ II GC-MS 质谱仪(Thermo Fisher, Austin, TX)和 6224 TOF LC/MS 质谱仪(Agilent Technologies, Santa Clara, CA); 熔点测定使用 Büchi M-545 数字熔点仪(未经校正). 柱 层析硅胶(200 300 目)和 TLC 薄层层析硅胶板均从青 岛海洋化工厂购买; 使用石油醚沸程为 $60 \sim 90{ }^{\circ} \mathrm{C}$, 其 他溶剂如无特殊说明均经标准操作干燥处理, 订购试剂 均为分析纯或化学纯. 中间体二芳基碘鎓四氟硼酸盐和 4,6-二甲氧基-2-甲砜基嘧啶分别参照文献方法合 成 ${ }^{[11,12]}$. 


\section{2 实验方法}

\subsection{1 中间体 2 的合成}

在 $1 \mathrm{~L}$ 三颈瓶中, 将 3,5-二羟基甲苯(1) $100 \mathrm{~g}$ 溶解 于 $400 \mathrm{~mL}$ 的丙三醇中, 反应温度加热至 $80{ }^{\circ} \mathrm{C}$, 并将 $\mathrm{CO}_{2}$ 通入反应体系中, 液面鼓泡速度控制于 2 次 $/ \mathrm{s}$, 反应 约 $12 \mathrm{~h}$, 停止反应并降温至室温, 将反应体系缓慢加入 至 $300 \mathrm{~mL}$ 的 $2 \mu \mathrm{mol} \cdot \mathrm{L}^{-1}$ 盐酸溶液中, 大量白色固体析 出，静置抽滤得到固体中间体 2 .

\subsection{2中间体 $\mathbf{3}$ 的合成}

$\mathrm{N}_{2}$ 保护下，在 $500 \mathrm{~mL}$ 三颈瓶中将 $100 \mathrm{mmol}$ 的 2,6二羟基-4-甲基苯甲酸(2)、 $1 \mathrm{mmol}$ DMAP 以及 $9.5 \mathrm{~mL}$ 丙 酮溶解至 $100 \mathrm{~mL}$ 干燥的乙二醇二甲醚中, 随后将体系 冷却至 $-10{ }^{\circ} \mathrm{C}$, 并向体系中缓慢滴加 $10.5 \mathrm{~mL}$ 二氯亚 砜和 $50 \mathrm{~mL}$ 乙二醇二甲醚混合溶液. 保持体系温度低于 $30{ }^{\circ} \mathrm{C}$, 持续反应约 $2 \mathrm{~d}$, 薄层色谱(TLC)监测直至原料 反应完全, 将体系中的混合溶液减压脱干, 大量固体析 出, 用饱和碳酸氢钠溶液对其进行多次洗涤, 剩余固体 用正己烷/乙酸乙酯重结晶即可得到纯度较高的关键中 间体 3, 产率 74\%, m.p. 105 106 ${ }^{\circ} \mathrm{C}$; ${ }^{1} \mathrm{H}$ NMR (600 MHz, DMSO- $\left.d_{6}\right) \delta: 10.21(\mathrm{~s}, 1 \mathrm{H}), 6.50(\mathrm{~s}, 1 \mathrm{H}), 6.40(\mathrm{~s}$, $1 \mathrm{H}), 2.28(\mathrm{~s}, 3 \mathrm{H}), 1.70(\mathrm{~s}, 6 \mathrm{H}) ;{ }^{13} \mathrm{C}$ NMR $(150 \mathrm{MHz}$, $\left.\mathrm{CDCl}_{3}\right) \delta: 165.2,160.9,155.2,149.9,111.1,107.9,106.9$, 96.7, 25.5, 22.3. EIMS $m / z: 208.00\left(\mathrm{M}^{+}\right)$. Anal. calcd for $\mathrm{C}_{11} \mathrm{H}_{12} \mathrm{O}_{4}$ : C 63.45, H 5.81; found C 63.66, H 5.79.

\subsection{3中间体 5 的合成}

在 $100 \mathrm{~mL}$ 单颈瓶中, 将 $5 \mathrm{mmol}$ 中间体 3 溶于 25 $\mathrm{mL}$ THF 或 $20 \mathrm{~mL}$ DMF 中, 加入 $5.5 \mathrm{mmol}$ 叔丁醇钾 (KTB)或 $\mathrm{K}_{2} \mathrm{CO}_{3}$, 控温于 $70{ }^{\circ} \mathrm{C}$, 向体系中加入 $5.5 \mathrm{mmol}$ 对应的二芳基碘鎓四氟硼酸盐或者邻(对)氟硝基苯, TLC 跟踪反应进程直至原料消失. 随后将 $6 \mathrm{mmol}$ $\mathrm{NaOH}$ 加入反应体系后, 将反应温度升温至 $60{ }^{\circ} \mathrm{C}$, TLC 跟踪反应进程直至中间态消失, 待反应体系冷却至室温 后, 加 $2 \mathrm{~mol} / \mathrm{L}$ 盐酸调节 $\mathrm{pH}$ 值为 1 , 固体析出, 低温静 置抽滤, 红外干燥得到中间体 $\mathbf{5}$, 无需进一步提纯可直 接投入下一步反应当中.

\subsection{4 目标化合物 $6 \mathrm{a} \sim 6 \mathrm{~m}$ 的合成}

在 $100 \mathrm{~mL}$ 单颈瓶中, 将 $2 \mathrm{mmol}$ 中间体 5 和 $8 \mathrm{mmol}$ 无水 $\mathrm{K}_{2} \mathrm{CO}_{3}$ 溶于 $40 \mathrm{~mL}$ 重蒸甲苯中, 室温搅拌约 $1 \mathrm{~h}$, 向 反应体系中加入 $2.2 \mathrm{mmol} 4,6$-二甲氧基-2-甲砜基嘧啶, 并将体系升温至甲苯回流, TLC 跟踪反应进程. 反应完 全后将体系冷却至室温, 加入 $40 \mathrm{~mL} 2 \mathrm{~mol} / \mathrm{L}$ 盐酸溶液 淬灭反应, 乙酸乙酯萃取 $(20 \mathrm{~mL} \times 3)$, 合并有机相, 有 机相用无水硫酸钠进行干燥, 减压蒸馏拌样, 经柱层析 分离提纯 $[V($ 石油醚 $) / V($ 丙酮 $)=20 / 10]$ 得到目标化合物.
2-[(4,6-二甲氧基嘧啶-2-基)氧基]-4-甲基-6-苯氧基 苯甲酸(6a): 产率 $75 \%$. 白色固体, m.p. $135 \sim 136{ }^{\circ} \mathrm{C} ;{ }^{1} \mathrm{H}$ NMR $\left(400 \mathrm{MHz}\right.$, DMSO- $\left.d_{6}\right) \delta: 12.91(\mathrm{~s}, 1 \mathrm{H}), 7.35(\mathrm{dd}$, $J=7.8,7.8 \mathrm{~Hz}, 2 \mathrm{H}), 7.10(\mathrm{dd}, J=7.2,7.2 \mathrm{~Hz}, 1 \mathrm{H}), 7.02 \sim$ $6.90(\mathrm{~m}, 3 \mathrm{H}), 6.68(\mathrm{~s}, 1 \mathrm{H}), 5.97(\mathrm{~s}, 1 \mathrm{H}), 3.79(\mathrm{~s}, 6 \mathrm{H}), 2.28$ (s, $3 \mathrm{H}) ;{ }^{13} \mathrm{C}$ NMR (100 MHz, DMSO- $\left.d_{6}\right) \delta: 172.2,164.6$, $163.1,156.8,153.4,149.8,141.3,129.6,123.0,118.6$, $118.6,117.8,116.8,83.9,54.2,20.9$. HRMS calcd for $\mathrm{C}_{20} \mathrm{H}_{18} \mathrm{~N}_{2} \mathrm{NaO}_{6}[\mathrm{M}+\mathrm{Na}]^{+}$405.1057, found 405.1060.

2-[(4,6-二甲氧基嘧啶-2-基)氧基]-6-(2-氟苯氧基)-4甲基苯甲酸 $(6 \mathbf{b})$ : 产率 78\%. 白色固体, m.p. 136 $137{ }^{\circ} \mathrm{C} ;{ }^{1} \mathrm{H}$ NMR (600 MHz, DMSO- $\left.d_{6}\right) \delta: 13.05(\mathrm{~s}, 1 \mathrm{H})$, $7.41 \sim 7.33(\mathrm{~m}, 1 \mathrm{H}), 7.23 \sim 7.16(\mathrm{~m}, 2 \mathrm{H}), 7.07$ (dd, $J=8.6$, $8.6 \mathrm{~Hz}, 1 \mathrm{H}), 6.96(\mathrm{~s}, 1 \mathrm{H}), 6.64(\mathrm{~s}, 1 \mathrm{H}), 6.01(\mathrm{~s}, 1 \mathrm{H}), 3.80$ $(\mathrm{s}, 6 \mathrm{H}), 2.28(\mathrm{~s}, 3 \mathrm{H}) ;{ }^{13} \mathrm{C}$ NMR $\left(100 \mathrm{MHz}\right.$, DMSO- $\left.d_{6}\right) \delta$ : 172.3, 164.6, 163.1, 153.5, 152.7 (d, $J=244.0 \mathrm{~Hz}), 149.8$, 143.3 (d, $J=11.0 \mathrm{~Hz}), 141.5,125.1$ (d, $J=7.0 \mathrm{~Hz}), 124.9$ (d, $J=7.0 \mathrm{~Hz}), 120.7,118.6,117.6,116.9(\mathrm{~d}, J=17.0 \mathrm{~Hz})$, 115.0, 83.9, 54.3, 21.0. HRMS calcd for $\mathrm{C}_{20} \mathrm{H}_{17} \mathrm{FN}_{2} \mathrm{NaO}_{6}$ $[\mathrm{M}+\mathrm{Na}]^{+}$423.0963, found 423.0964 .

2-[(4,6-二甲氧基嘧啶-2-基)氧基]-6-(3-氟苯氧基)-4甲基苯甲酸(6c): 产率 66\%. 白色固体，m.p. 130 $131{ }^{\circ} \mathrm{C} ;{ }^{1} \mathrm{H}$ NMR $\left(600 \mathrm{MHz}\right.$, DMSO- $\left.d_{6}\right) \delta: 13.03(\mathrm{~s}, 1 \mathrm{H})$, 7.39 (dd, $J=15.6,8.4 \mathrm{~Hz}, 1 \mathrm{H}), 7.04$ (s, 1H), 6.95 (dd, $J=$ $7.8,7.8 \mathrm{~Hz}, 1 \mathrm{H}), 6.85(\mathrm{~s}, 1 \mathrm{H}), 6.82 \sim 6.76(\mathrm{~m}, 2 \mathrm{H}), 6.00(\mathrm{~s}$, $1 \mathrm{H}), 3.79(\mathrm{~s}, 6 \mathrm{H}), 2.32(\mathrm{~s}, 3 \mathrm{H}) ;{ }^{13} \mathrm{C}$ NMR $(100 \mathrm{MHz}$, DMSO- $\left.d_{6}\right) \delta: 172.2,164.5,163.1,162.4(\mathrm{~d}, J=242.0 \mathrm{~Hz})$, $158.6(\mathrm{~d}, J=11.0 \mathrm{~Hz}), 152.4,149.9,141.9,130.9$ (d, $J=$ $10.0 \mathrm{~Hz}$ ), 119.6, 119.0, 118.0, 113.2, 109.6 (d, $J=21.0$ $\mathrm{Hz}), 104.9$ (d, $J=24.0 \mathrm{~Hz}), 84.0,54.2$, 20.91. HRMS calcd for $\mathrm{C}_{20} \mathrm{H}_{17} \mathrm{FN}_{2} \mathrm{NaO}_{6}[\mathrm{M}+\mathrm{Na}]^{+}$423.0963, found 423.0963 .

2-[(4,6-二甲氧基嘧啶-2-基)氧基]-6-(4-氟苯氧基)-4甲基苯甲酸(6d): 产率 85\%. 白色固体，m.p. 143 $144{ }^{\circ} \mathrm{C} ;{ }^{1} \mathrm{H}$ NMR (600 MHz, DMSO- $\left.d_{6}\right) \delta: 13.02(\mathrm{~s}, 1 \mathrm{H})$, 7.23 (dd, $J=9.0,9.0 \mathrm{~Hz}, 2 \mathrm{H}), 7.03(\mathrm{dd}, J=9.0,4.2 \mathrm{~Hz}$, 2H), $6.96(\mathrm{~s}, 1 \mathrm{H}), 6.68(\mathrm{~s}, 1 \mathrm{H}), 6.01(\mathrm{~s}, 1 \mathrm{H}), 3.80(\mathrm{~s}, 6 \mathrm{H})$, $2.28(\mathrm{~s}, 3 \mathrm{H}) ;{ }^{13} \mathrm{C}$ NMR $\left(100 \mathrm{MHz}\right.$, DMSO- $\left.d_{6}\right) \delta: 172.3$, 164.7, 163.1, 157.8 (d, $J=237.0 \mathrm{~Hz}), 153.8,152.9,149.8$, $141.5,119.8$ (d, $J=8.0 \mathrm{~Hz}), 118.5$ (d, $J=22.0 \mathrm{~Hz}), 116.4$, 116.2, 83.9, 54.3, 21.0. HRMS calcd for $\mathrm{C}_{20} \mathrm{H}_{17} \mathrm{FN}_{2} \mathrm{NaO}_{6}$ $[\mathrm{M}+\mathrm{Na}]^{+}$423.0963, found 423.0961 .

2-(2-氯苯氧基)-6-[(4,6-二甲氧基嘧啶-2-基)氧基]-4甲基苯甲酸(6e): 产率 68\%. 白色固体, m.p. 138 $139{ }^{\circ} \mathrm{C} ;{ }^{1} \mathrm{H}$ NMR (400 MHz, DMSO- $\left.d_{6}\right) \delta: 13.02(\mathrm{~s}, 1 \mathrm{H})$, $7.54(\mathrm{~d}, J=7.8 \mathrm{~Hz}, 1 \mathrm{H}), 7.31(\mathrm{dd}, J=7.6,7.6 \mathrm{~Hz}, 1 \mathrm{H})$, 
$7.15(\mathrm{dd}, J=7.6,7.6 \mathrm{~Hz}, 1 \mathrm{H}), 7.04 \sim 6.89(\mathrm{~m}, 2 \mathrm{H}), 6.61(\mathrm{~s}$, $1 \mathrm{H}), 5.98(\mathrm{~s}, 1 \mathrm{H}), 3.79(\mathrm{~s}, 6 \mathrm{H}), 2.28(\mathrm{~s}, 3 \mathrm{H}) ;{ }^{13} \mathrm{C} \mathrm{NMR}$ $\left(100 \mathrm{MHz}, \mathrm{DMSO}-d_{6}\right) \delta: 172.3,164.5,163.1,153.0,152.0$, $149.9,141.6,130.4,128.4,124.7,123.4,119.5,118.9$, $118.1,115.9,84.0,54.3,21.0$. HRMS calcd for $\mathrm{C}_{20} \mathrm{H}_{17} \mathrm{Cl}-$ $\mathrm{N}_{2} \mathrm{NaO}_{6}[\mathrm{M}+\mathrm{Na}]^{+}$439.0667, found 439.0665.

2-(3-氯苯氧基)-6-[(4,6-二甲氧基嘧啶-2-基)氧基]-4甲基苯甲酸(6f): 产率 $67 \%$. 白色固体，m.p. 135 $136{ }^{\circ} \mathrm{C} ;{ }^{1} \mathrm{H}$ NMR (600 MHz, DMSO- $\left.d_{6}\right) \delta: 13.03$ (s, 1H), $7.39(\mathrm{dd}, J=7.8,7.8 \mathrm{~Hz}, 1 \mathrm{H}), 7.16(\mathrm{~d}, J=7.8 \mathrm{~Hz}, 1 \mathrm{H})$, $7.05(\mathrm{~s}, 1 \mathrm{H}), 6.98 \sim 6.90(\mathrm{~m}, 2 \mathrm{H}), 6.86(\mathrm{~s}, 1 \mathrm{H}), 6.00(\mathrm{~s}$, 1H), 3.79 (s, 6H), $2.33(\mathrm{~s}, 3 \mathrm{H}) ;{ }^{13} \mathrm{C} \mathrm{NMR}(100 \mathrm{MHz}$, DMSO- $\left.d_{6}\right) \delta: 172.2,164.4,163.0,158.3,152.3,149.9$, $142.1,133.5,131.0,122.6,119.7,119.1,118.3,117.1$, 115.9, 84.0, 54.2, 20.9. HRMS calcd for $\mathrm{C}_{20} \mathrm{H}_{17} \mathrm{ClN}_{2} \mathrm{NaO}_{6}$ $[\mathrm{M}+\mathrm{Na}]^{+}$439.0667, found 439.0668 .

2-(4-氯苯氧基)-6-[(4,6-二甲氧基嘧啶-2-基)氧基]-4甲基苯甲酸 $(6 \mathrm{~g})$ : 产率 $81 \%$. 白色固体，m.p. 152 $153{ }^{\circ} \mathrm{C} ;{ }^{1} \mathrm{H}$ NMR (600 MHz, DMSO- $\left.d_{6}\right) \delta: 13.03$ (s, 1H), $7.42(\mathrm{~d}, J=9.0 \mathrm{~Hz}, 2 \mathrm{H}), 7.06 \sim 6.94(\mathrm{~m}, 3 \mathrm{H}), 6.78(\mathrm{~s}, 1 \mathrm{H})$, $6.00(\mathrm{~s}, 1 \mathrm{H}), 3.79(\mathrm{~s}, 6 \mathrm{H}), 2.30(\mathrm{~s}, 3 \mathrm{H}) ;{ }^{13} \mathrm{C}$ NMR $(100$ $\left.\mathrm{MHz}, \mathrm{DMSO}-d_{6}\right) \delta: 172.3,164.6,163.1,156.0,152.9$, $149.9,141.8,129.5,126.8,119.3,118.8,117.5,84.0,54.3$, 20.9. HRMS calcd for $\mathrm{C}_{20} \mathrm{H}_{17} \mathrm{ClN}_{2} \mathrm{NaO}_{6}[\mathrm{M}+\mathrm{Na}]^{+}$ 439.0667, found 439.0669 .

2-[(4,6-二甲氧基嘧啶-2-基)氧基]-4-甲基-6-(2-硝基 苯氧基)苯甲酸 $(6 \mathrm{~h})$ : 产率 76\%. 白色固体, m.p. 163 $164{ }^{\circ} \mathrm{C}$; ${ }^{1} \mathrm{H}$ NMR (600 MHz, DMSO- $\left.d_{6}\right) \delta: 13.03$ (s, 1H), $7.58(\mathrm{~d}, J=8.0 \mathrm{~Hz}, 1 \mathrm{H}), 7.34(\mathrm{dd}, J=7.8,7.8 \mathrm{~Hz}, 1 \mathrm{H})$, $7.18(\mathrm{dd}, J=7.8,7.8 \mathrm{~Hz}, 1 \mathrm{H}), 7.03 \sim 6.94(\mathrm{~m}, 2 \mathrm{H}), 6.00(\mathrm{~s}$, $1 \mathrm{H}), 3.80(\mathrm{~s}, 6 \mathrm{H}), 2.28(\mathrm{~s}, 3 \mathrm{H}) ;{ }^{13} \mathrm{C} \mathrm{NMR}(100 \mathrm{MHz}$, DMSO- $\left.d_{6}\right) \delta: 172.6,164.4,163.3,152.2,150.4,150.2$, $142.5,140.1,134.8,125.5,123.4,120.3,119.1,118.8$, 118.1, 84.1, 54.2, 54.1, 20.8. HRMS calcd for $\mathrm{C}_{20} \mathrm{H}_{17} \mathrm{~N}_{3}-$ $\mathrm{NaO}_{8}[\mathrm{M}+\mathrm{Na}]^{+}$450.0908, found 450.0906.

2-[(4,6-二甲氧基嘧啶-2-基)氧基]-4-甲基-6-(4-硝基 苯氧基)苯甲酸(6i): 产率 $68 \%$. 白色固体, m.p. 148 $149{ }^{\circ} \mathrm{C} ;{ }^{1} \mathrm{H}$ NMR (600 MHz, DMSO- $\left.d_{6}\right) \delta: 13.12$ (s, 1H), $8.26(\mathrm{~d}, J=8.4 \mathrm{~Hz}, 2 \mathrm{H}), 7.15$ (s, 1H), 7.10 (d, $J=8.4 \mathrm{~Hz}$, 2H), 7.02 (s, 1H), 6.00 (s, 1H), 3.79 (s, 6H), 2.37 (s, 3H); ${ }^{13} \mathrm{C}$ NMR (100 MHz, DMSO- $d_{6}$ ) $\delta$ : 172.3, 164.3, 163.1, $163.0,151.3,150.3,142.7,142.0,125.9,120.8,119.4$, $116.8,109.3,84.1,54.3,20.9$. HRMS calcd for $\mathrm{C}_{20} \mathrm{H}_{17} \mathrm{~N}_{3}-$ $\mathrm{NaO}_{8}[\mathrm{M}+\mathrm{Na}]^{+}$450.0908, found 450.0910.

2-[(4,6-二甲氧基嘧啶-2-基)氧基]-4-甲基-6-(3-甲基- 4-硝基苯氧基)苯甲酸(6j)：产率 69\% . 白色固体，m.p. $151 \sim 152{ }^{\circ} \mathrm{C} ;{ }^{1} \mathrm{H}$ NMR (400 MHz, DMSO- $d_{6}$ ) $\delta: 13.08$ (s, $1 \mathrm{H}), 8.06(\mathrm{~d}, J=9.0 \mathrm{~Hz}, 1 \mathrm{H}), 7.11(\mathrm{~s}, 1 \mathrm{H}), 7.01(\mathrm{~s}, 1 \mathrm{H})$, $6.97(\mathrm{~s}, 1 \mathrm{H}), 6.88(\mathrm{~d}, J=9.0 \mathrm{~Hz}, 1 \mathrm{H}), 5.97(\mathrm{~s}, 6 \mathrm{H}), 2.53$ (s, $3 \mathrm{H}), 2.36(\mathrm{~s}, 3 \mathrm{H}) ;{ }^{13} \mathrm{C} \mathrm{NMR}\left(100 \mathrm{MHz}, \mathrm{DMSO}-d_{6}\right) \delta$ : $172.2,164.2,163.0,161.1,151.4,150.2,143.0,142.5$, 136.2, 127.2, 120.5, 119.8, 119.3, 119.1, 114.4, 84.1, 54.3, 20.9, 20.5. HRMS calcd for $\mathrm{C}_{21} \mathrm{H}_{19} \mathrm{~N}_{3} \mathrm{NaO}_{8}[\mathrm{M}+\mathrm{Na}]^{+}$ 464.1064, found 464.1061.

2-[(4,6-二甲氧基嘧啶-2-基)氧基]-4-甲基-6-(2-甲 基-4-硝基苯氧基)苯甲酸(6k)：产率 54\%. 白色固体， m.p. $175 \sim 176{ }^{\circ} \mathrm{C} ;{ }^{1} \mathrm{H}$ NMR $\left(600 \mathrm{MHz}, \mathrm{DMSO}-d_{6}\right) \delta$ : $13.06(\mathrm{~s}, 1 \mathrm{H}), 8.22(\mathrm{~s}, 1 \mathrm{H}), 8.07(\mathrm{dd}, J=9.0,1.8 \mathrm{~Hz}, 1 \mathrm{H})$, $7.11(\mathrm{~s}, 1 \mathrm{H}), 6.94(\mathrm{~s}, 1 \mathrm{H}), 6.79$ (d, $J=9.0 \mathrm{~Hz}, 1 \mathrm{H}), 5.99$ (s, $1 \mathrm{H}), 3.79(\mathrm{~s}, 6 \mathrm{H}), 2.36(\mathrm{~s}, 3 \mathrm{H}), 2.35(\mathrm{~s}, 3 \mathrm{H}) ;{ }^{13} \mathrm{C} \mathrm{NMR}$ $\left(100 \mathrm{MHz}, \mathrm{DMSO}-d_{6}\right) \delta: 172.4,164.4,163.3,161.2,152.0$, $150.5,142.6,141.9,128.6,126.3,123.3,120.4,118.9$, 115.3, 54.4, 21.1, 16.1. HRMS calcd for $\mathrm{C}_{21} \mathrm{H}_{19} \mathrm{~N}_{3} \mathrm{NaO}_{8}$ $[\mathrm{M}+\mathrm{Na}]^{+}$464.1064, found 464.1063.

2-[(4,6-二甲氧基嘧啶-2-基)氧基]-6-(2-氟-4-硝基苯 氧基)-4-甲基苯甲酸(6I): 产率 71\%. 白色固体，m.p. $145 \sim 146{ }^{\circ} \mathrm{C} ;{ }^{1} \mathrm{H}$ NMR (600 MHz, DMSO- $\left.d_{6}\right) \delta: 13.17$ (s, $1 \mathrm{H}), 8.33(\mathrm{~d}, J=10.2 \mathrm{~Hz}, 1 \mathrm{H}), 8.10$ (d, $J=8.4 \mathrm{~Hz}, 1 \mathrm{H})$, $7.15(\mathrm{~s}, 1 \mathrm{H}), 7.06(\mathrm{~s}, 1 \mathrm{H}), 7.05 \sim 7.00(\mathrm{~m}, 1 \mathrm{H}), 3.78(\mathrm{~s}$, $6 \mathrm{H}), 2.36(\mathrm{~s}, 3 \mathrm{H}) ;{ }^{13} \mathrm{C}$ NMR (100 MHz, DMSO-d $) \delta$ : 172.2, 164.0, 162.9, 151.7, 150.9 (d, $J=10.0 \mathrm{~Hz}), 150.5$ (d, $J=248.0 \mathrm{~Hz}), 150.3,142.7,141.8$ (d, $J=10.0 \mathrm{~Hz})$, $121.1(\mathrm{~d}, \quad J=29.0 \mathrm{~Hz}), 118.7,118.6,117.6,112.9(\mathrm{~d}, J=$ $23.0 \mathrm{~Hz}), 84.1,54.3,20.9$. HRMS calcd for $\mathrm{C}_{20} \mathrm{H}_{16} \mathrm{FN}_{3}-$ $\mathrm{NaO}_{8}[\mathrm{M}+\mathrm{Na}]^{+}$468.0814, found 468.0829.

2-(2-氯-4-硝基苯氧基)-6-[(4,6-二甲氧基嘧啶-2-基) 氧基]-4-甲基苯甲酸(6m): 产率 64\%. 白色固体，m.p. $150 \sim 151{ }^{\circ} \mathrm{C} ;{ }^{1} \mathrm{H}$ NMR (600 MHz, DMSO- $\left.d_{6}\right) \delta: 13.16(\mathrm{~s}$, $1 \mathrm{H}), 8.45(\mathrm{~d}, J=2.4 \mathrm{~Hz}, 1 \mathrm{H}), 8.20(\mathrm{dd}, J=9.0,2.4 \mathrm{~Hz}$, 1H), 7.17 (s, 1H), 7.05 (s, 1H), 6.93 (d, $J=9.2 \mathrm{~Hz}, 1 \mathrm{H})$, 5.99 (s, 1H), $3.78(\mathrm{~s}, 6 \mathrm{H}), 2.37$ (s, 3H); ${ }^{13} \mathrm{C}$ NMR $(100$ $\left.\mathrm{MHz}, \mathrm{DMSO}-d_{6}\right) \delta: 172.2,163.9,163.0,158.5,150.9$, $150.3,142.8,142.0,125.8,124.2,122.3,121.1,119.0$, 119.0, 116.5, 84.1, 54.3, 20.9. HRMS calcd for $\mathrm{C}_{20} \mathrm{H}_{16} \mathrm{Cl}-$ $\mathrm{N}_{3} \mathrm{NaO}_{8}[\mathrm{M}+\mathrm{Na}]^{+}$484.0518, found 484.0524.

\section{2 .5 酶抑制活性测定实验}

首先将化合物配制四种不同浓度的溶液，即母液的 浓度为 $6 、 60 、 600 、 6000 \mu \mathrm{mol} \cdot \mathrm{L}^{-1}$ ，反应时再进行稀释， 在反应体系中的最终浓度为 $0.2 、 2 、 20 、 200 \mu \mathrm{mol} \cdot \mathrm{L}^{-1}$. 使用的野生型 AHAS 为拟南芥 AHAS 催化亚基表达蛋 
白质裂解液. P197L 突变体采用 Megaprimer PCR 定点突 变的方法, 以含有拟南芥 AHAS 基因的质粒 $\mathrm{pET} 28 \mathrm{a}$ $(+)-A C S U: T 86$ 作为模板, 进行定点突变, 得到含有突 变位点的产物 ${ }^{[13]}$. 突变产物经过限制性内切酶酶切克 隆到所要连接的载体中. 把经过测序验证的突变质粒转 化到 E. coli (XL1-blue)中进行酶表达.

活性结果测定公式: $V_{\mathrm{i}}=V_{\infty}+\left(V_{0}-V_{\infty}\right) /\left(1+[\mathrm{I}] / K_{\mathrm{i}}\right)$

[I]代表所加化合物样品的浓度; $K_{\mathrm{i}}$ 代表样品的抑制 率为 $50 \%$ 时所对应的测试样品浓度. $V_{\mathrm{i}}$ 和 $V_{0}$ 分别为加抑 制剂和未加抑制剂的酶反应速率; $V_{\mathrm{i}}$ 数值用 residual activity (百分比)表示; $V_{0}$ 设为 $100 \%, V_{\infty}$ 表示在饱和浓度 的抑制浓度下的残留活性.

\section{2 .6 模型构建与分子对接}

从 Protein Data Bank 数据库中下载野生型 AtAHAS 的 $5 \mathrm{~K} 2 \mathrm{O}$ 晶体结构作为受体 ${ }^{[14]}$. 采用 SYBYL 2.0 中的生 物聚合物突变建模工具, 对 P197L 突变体进行构建. 随 后, 采用 Powell 法 ${ }^{[15]}$ 对突变位点周围 $3 \AA$ 距离内的残基 进行 3000 步的优化, 收玫条件设置为 $42 \mathrm{~J} / \mathrm{mol}$. 采用 Auto Dock 4.0 程序进行分子对接, 活性腔中心坐标设为 $x=77.68, y=75.62, z=80.86$, 格点大小为 $50 \times 50 \times 50$, 步长设为 $0.375 \AA$. 设置每个小分子化合物产生 256 个 构象, 其它参数设为默认值 ${ }^{[16]}$. 最后, 对选择的构象进 行 1-2ns 的平衡动力学模拟.

\subsection{7 温室盆栽除草活性测定实验}

供试靶标为浙江省化工研究院提供的敏感生物型 播娘蒿和抗性(P197L-AHAS)播娘蒿 ${ }^{[17]}$. 播娘蒿种子用 $0.05 \% \mathrm{GA}_{3}$ 浸种 $24 \mathrm{~h}$, 打破休眠, 再用清水洗净后播种. 取内径 $7.5 \mathrm{~cm}$ 花盆, 装复合营养土至 $3 / 4$ 处, 均匀覆土 $0.2 \mathrm{~cm}$, 人工气候室中保持温度 $15{ }^{\circ} \mathrm{C}$ (夜) $/ 25{ }^{\circ} \mathrm{C}$ (昼). 子叶展开后, 将苗搬至温室进行炼苗, 待杂草长至 4 叶 期进行备用. 各化合物按照 $150 \mathrm{~g}$ ai/ha 剂量用自动喷雾 塔(型号: 3WPSH-700E)给杂草施药后, 将其移入人工气 候室, $35 \mathrm{~d}$ 后调查其对杂草的活性(生长抑制率, \%).

\section{辅助材料(Supporting Information) 化合物 $6 \mathrm{a} \sim 6 \mathrm{~m}$ 的} ${ }^{1} \mathrm{H}$ NMR 和 ${ }^{13} \mathrm{C}$ NMR 谱图. 这些材料可以免费从本刊网 站(http://s10oc-journal.cn/)上下载.

\section{References}

[1] (a) Bar-Ilan, A.; Balan, V.; Tittmann, K.; Golbik, R.; Vyazmensky, M.; Huebner, G.; Barak, Z.; Chipman, D. M. Biochemistry 2001, 40, 11946.

(b) Dug gleby, R. G.; Pang, S. S. J. Biochem. Mol. Biol. 2000, 33, 1 .

[2] McCourt, J. A.; Pang, S. S.; King-Scott, J.; Guddat, L. W.; Duggleby, R. G.. Proc. Natl. Acad. Sci. U. S. A. 2006, 103, 569.

[3] (a) Yu, Q.; Powles, S. B. Pest Manage. Sci. 2014, 70, 1340. (b) Tranel, P. J.; Wright, T. R. Weed Sci. 2002, 50, 700.

[4] Heap, I. The International Survey of Herbicide-Resistant Weeds, www. weedscience. org (accessed December 29, 2018).

[5] (a) Duggleby, R. G.; Pang, S. S.; Yu, H.; Guddat, L. W. Eur. J. Biochem. 2003, 270, 2895.

(b) Hamouzova, K.; Kosnarova, P.; Salava, J.; Soukup, J.; Hamouz, P. Pest Manage. Sci. 2014, 70, 541.

(c) Beckie, H. J.; Warwick, S. I.; Sauder, C. A.; Lozinski, C.; Shirriff, S. Weed Technol. 2011, 25, 170.

[6] (a) Legere, A.; Stevenson, F. C.; Beckie, H. J.; Warwick, S. I.; Johnson, E. N.; Hrynewich, B.; Lozinski, C. Weed Sci. 2013, 61, 267.

(b) Intanon, S.; Perez-Jones, A.; Hulting, A. G.; Mallory-Smith, C. A. Weed Sci. 2011, 59, 431

[7] Sibony, M.; Michel, A.; Haas, H. U.; Rubin, B.; Hurle, K. Weed Res. 2001, 41, 509.

[8] (a) Ji, F. Q.; Niu, C. W.; Chen, C. N.; Chen, Q.; Yang, G. F.; Xi, Z.; Zhan, C. G. ChemMedChem 2008, 3, 1203.

(b) Liu, Y. C.; Qu, R. Y.; Chen, Q.; Yang, J. F.; Niu, C. W.; Zhen, X.; Yang, G. F. J. Agric. Food Chem. 2016, 64, 4845.

(c) Qu, R. Y.; Yang, J. F.; Liu, Y. C.; Chen, Q.; Hao, G. F.; Niu, C. W.; Xi, Z.; Yang, G. F. Pest Manage. Sci. 2017, 73, 1373.

(d) Qu, R. Y.; Yang, J. F.; Ponnam D.; Kang, W. M.; Liu, Y. C.; Chen, Q.; Niu, C. W.; Zhen, X.; Yang, G. F. J. Agric. Food Chem. 2017, 65, 11170 .

[9] Li, K. J.; Qu, R. Y.; Liu, Y. C.; Yang, J. F.; Ponnam D.; Chen, Q.; Niu, C. W.; Zhen, X.; Yang, G. F. J. Agric. Food Chem. 2018, 66, 3773.

[10] Qu, R. Y.; Liu, Y. C.; Wu, Q. Y.; Chen, Q.; Yang, G. F. Tetrahedron 2015, 71, 8123 .

[11] Chan, L.; McNally, A.; Toh, Q. Y.; Mendoza, A.; Gaunt, M. J. Chem. Sci. 2015, 6, 1277.

[12] Bolli, M. H.; Marfurt, J.; Grisostomi, C.; Boss, C.; Binkert, C.; Hess, P.; Treiber, A.; Thorin, E.; Morrison, K.; Buchmann, S.; Bur, D.; Ramuz, H.; Clozel, M.; Fischli, W.; Weller, T. J. Med. Chem. 2004, 47, 2776.

[13] Singh, B. K.; Stidham, M. A.; Shaner, D. L. Anal. Biochem. 1988, 171,173 .

[14] Garcia, M. D.; Nouwens, A.; Lonhienne, T. G.; Guddat, L. W. Proc. Natl. Acad. Sci. U. S. A. 2017, 114, 1091.

[15] Powell, M. J. D. Math. Program. 1977, 12, 241

[16] Morris, G. M.; Goodsell, D. S.; Halliday, R. S.; Huey, R.; Hart, W. E.; Belew, R. K.; Olson, A. J. Comput. Chem. 1998, 19, 1639.

[17] Cui, H. L.; Zhang, C. X.; Wei, S. H.; Zhang, H. J.; Li, X. J.; Zhang, Y. Q.; Wang, G. Q. Weed Sci. 2011, 59, 376. 